\title{
SPECT-derived absolute myocardial perfusion measures: A step in the right direction
}

\author{
Navkaranbir S. Bajaj, MD, MPH, ${ }^{\mathrm{a}, \mathrm{b}, \mathrm{c}}$ and Pradeep Bhambhvani, MD $^{\mathrm{c}}$ \\ a Division of Cardiovascular Disease, University of Alabama at Birmingham, Birmingham, AL \\ b Section of Cardiology, Birmingham Veterans Affair Medical Center, Birmingham, AL \\ c Division of Molecular Imaging and Therapeutics, Department of Radiology, University of \\ Alabama at Birmingham, Birmingham, AL
}

Received Nov 18, 2019; accepted Nov 18, 2019

doi: $10.1007 / \mathrm{s} 12350-019-01972-\mathrm{w}$

\section{See related article, pp. 1906-1918}

The traditional imaging concept of regional blood flow analysis by comparing best perfused areas to those that are not as well perfused is imperfect due to its relative nature. ${ }^{1}$ Quantification of absolute myocardial blood flow (MBF) at stress and rest, and the derivation of myocardial flow reserve (MFR) adds incremental diagnostic utility, powerful prognostic nature, and improved accuracy to relative perfusion imaging by positron emission tomography (PET). ${ }^{1-6}$ Consensus statement from the American Society of Nuclear Cardiology and the Society of Nuclear Medicine and Molecular Imaging Cardiovascular Council indicates that these PET-derived measures are at the cusp of translation into clinical practice. ${ }^{5}$ However, out of more than 6 million myocardial perfusion studies done annually in North America, greater than 99\% are performed with SPECT cameras, given the favorable economics, lack of cyclotron availability and expertise for PET imaging. ${ }^{7}$ Given the wide spread availability of SPECT cameras and favorable economics, it is but natural that attempts have been made over the past several years to determine absolute MBF measures with SPECT imaging. ${ }^{8}$

Due to limited sensitivity and lower temporal resolution of traditional SPECT cameras compared with

Reprint requests: Pradeep Bhambhvani, MD, Division of Molecular Imaging and Therapeutics, Department of Radiology, University of Alabama at Birmingham, 619 19th Street South, JT 777, Birmingham, AL 35249, USA; phambhvani@uabmc.edu

J Nucl Cardiol 2021;28:1919-22.

$1071-3581 / \$ 34.00$

Copyright (C) 2019 American Society of Nuclear Cardiology.
PET, measurement of absolute MBF has been challenging. Despite these difficulties, attempts have been made to ascertain the feasibility of dynamic acquisitions with conventional SPECT cameras. ${ }^{8,9}$ With the advent of solid-state detector (e.g., cadmium zinc telluride) cameras, the sensitivity, spatial, temporal, and energy resolution of SPECT imaging have improved dramatically. $^{10,11}$ These improvements in SPECT camera technology has made dynamic acquisition, a requisite for ascertainment of absolute MBF easier. ${ }^{7,8}$ Animal and human studies have demonstrated that solid-state detector SPECT cameras can measure MBF using either ${ }^{201}$ Thallium or ${ }^{99 \mathrm{~m}}$ Technetium $(99 \mathrm{mTc})$-based standard perfusion tracers which correlate with myocardial flow measured with microspheres and PET tracers. ${ }^{7,12}$

The current study by Giubbini et $\mathrm{l}^{13}$ shows reasonable correlation between MBF and MFR measured by N13NH3-PET and 99mTc-tetrofosmin CZT SPECT and builds on the previous studies ${ }^{7,8,14,15}$ outlined in the Table 1 which have compared the accuracy of SPECTderived MBF measures to PET measures. The study by Giubinni et al is the largest and also highlights the importance of motion and attenuation correction for accurate determination of MBF measures. Similar optimization of dynamic images was also performed in the study by Wells et $\mathrm{al}^{7}$ to obtain accurate MBF values.

While reviewing this study and others, we noticed several sources of variability including different PET and SPECT cameras, stress agents, stress and rest protocols, radiotracers, application of motion and attenuation correction, reconstruction techniques, and software packages. All studies noted correlation with PET measures but no systematic directional differences were noted in SPECT MBF or MFR as compared to PET measures. 
Table 1. Sources of variability in MBF and MFR determination with SPECT and PET across studies

\begin{tabular}{|c|c|c|c|c|c|}
\hline Study & $\begin{array}{l}\text { Giubbini } \\
\text { et al } \\
(2019)\end{array}$ & $\begin{array}{l}\text { Waterday } \\
\text { study } \\
\text { (2018) }\end{array}$ & $\begin{array}{c}\text { Wells et al } \\
(2017)\end{array}$ & $\begin{array}{c}\text { Nkoulou } \\
\text { et al }{ }^{14}(2016)\end{array}$ & Ito et $\mathrm{al}^{8}(2003)$ \\
\hline $\mathrm{N}$ & 54 & 30 & 31 & 32 & 29 \\
\hline $\begin{array}{c}\text { Mean age } \\
\text { (years) }\end{array}$ & 69 & 65 & 64 & 64 & 57 \\
\hline Mean BMI & _ & - (8\% obese) & 27.9 & 28 & - \\
\hline CAD (\%) & $26 \% *$ & $100 \%$ & - & - & $69 \%$ \\
\hline \multicolumn{6}{|c|}{ SPECT characteristics } \\
\hline $\begin{array}{l}\text { SPECT } \\
\text { Tracer }\end{array}$ & $\begin{array}{l}{ }^{99 \mathrm{~m}} \mathrm{Tc}- \\
\text { tetrofosmin }\end{array}$ & $\begin{array}{l}{ }^{99 \mathrm{~m}} \mathrm{Tc}- \\
\text { sestamibi }\end{array}$ & ${ }^{99 \mathrm{~m}}$ Tc-tetrofosmin & ${ }^{99 m}$ Tc-tetrofosmin & ${ }^{99 m}$ Tc-sestamibi \\
\hline $\begin{array}{l}\text { SPECT } \\
\text { camera }\end{array}$ & GE-CZT & D-SPECT & GE-CZT & GE-CZT & $\begin{array}{l}\text { Non CZT dual head } \\
\text { camera (ADAC } \\
\text { Labs) }\end{array}$ \\
\hline $\begin{array}{l}\text { Attenuation } \\
\text { correction } \\
\text { with CT }\end{array}$ & $\begin{array}{l}\text { Both } A C \text { and } \\
\text { non-AC }\end{array}$ & No & $\begin{array}{l}\text { Both AC and non- } \\
\text { AC }\end{array}$ & Yes & No \\
\hline $\begin{array}{l}\text { Dose SPECT } \\
\text { tracer }\end{array}$ & $\begin{array}{l}185 \mathrm{MBq} \text { after } \\
\text { stress } \\
370 \mathrm{MBq} \text { after } \\
\text { rest }\end{array}$ & $\begin{array}{l}3 \mathrm{MBq} / \mathrm{kg} \text { for } \\
\text { rest and } 9 \\
\mathrm{MBq} / \mathrm{kg} \\
\text { stress }\end{array}$ & $\begin{array}{l}316 \pm 71 \mathrm{MBq} \text { at } \\
\text { rest and then } \\
1122 \pm 170 \\
\mathrm{MBq} \text { at stress }\end{array}$ & $\begin{array}{l}330 \mathrm{MBq} \text { at stress } \\
\text { and } 3 \text { times rest } \\
\text { dose }\end{array}$ & $\begin{array}{c}370 \mathrm{MBq} \text { at rest and } \\
370 \mathrm{Mbq} \text { at stress }\end{array}$ \\
\hline Stressor & Regadenoson & Regadenoson & Dipyridamole & Adenosine & Adenosine \\
\hline Protocol & $\begin{array}{l}\text { Stress first same } \\
\text { day }\end{array}$ & $\begin{array}{l}\text { Rest first same } \\
\text { day }\end{array}$ & $\begin{array}{l}\text { Rest first same } \\
\text { day }\end{array}$ & $\begin{array}{l}\text { Stress first same } \\
\text { day }\end{array}$ & $\begin{array}{l}\text { Rest first } 2 \text { day } \\
\text { protocol }\end{array}$ \\
\hline Software & $\begin{array}{l}\text { Corridor-4DM- } \\
\text { Reserve } \\
\text { (v2015; } \\
\text { INVIA, Ann } \\
\text { Arbor, MI, } \\
\text { USA) }\end{array}$ & $\begin{array}{l}\text { Corridor 4DM } \\
\text { (v2015, } \\
\text { INVIA, Ann } \\
\text { Arbor, MI, } \\
\text { USA) }\end{array}$ & FlowQuant & $\begin{array}{l}\text { PMOD software } \\
\text { package } \\
\text { (version 3.1; } \\
\text { PMOD } \\
\text { Technologies } \\
\text { Ltd.) }\end{array}$ & Not reported \\
\hline \multicolumn{6}{|c|}{ PET characteristics } \\
\hline $\begin{array}{l}\text { Comparison } \\
\text { PET tracer }\end{array}$ & ${ }^{13} \mathrm{~N}-\mathrm{NH}_{3}$ & ${ }^{15} \mathrm{O}-\mathrm{H}_{2} \mathrm{O}$ & ${ }^{82} \mathrm{Rb}$ or ${ }^{13} \mathrm{~N}-\mathrm{NH}_{3}$ & ${ }^{13} \mathrm{~N}-\mathrm{NH}_{3}$ & ${ }^{15} \mathrm{O}-\mathrm{H}_{2} \mathrm{O}$ \\
\hline PET camera & $\begin{array}{l}\text { GE discovery } \\
690\end{array}$ & $\begin{array}{l}\text { GE discovery } \\
\text { VCT/RX }\end{array}$ & GE discovery 690 & $\begin{array}{l}\text { GE discovery ST/ } \\
\text { RX and } \\
\text { Discovery VCT }\end{array}$ & $\begin{array}{c}\text { Siemens-ECAT } \\
\text { EXACT HR+ }\end{array}$ \\
\hline $\begin{array}{l}\text { Dose of PET } \\
\text { tracer }\end{array}$ & $\begin{array}{l}\text { Bolus } 370 \mathrm{MBq} \\
\text { at stress } \\
\text { Rest not } \\
\text { reported }\end{array}$ & $\begin{array}{l}\text { 1.5-3 } \mathrm{MBq} / \mathrm{kg} \\
\text { at rest } \\
\text { followed by } \\
\text { same dose } \\
\text { for stress }\end{array}$ & $\begin{array}{l}10 \mathrm{MBq} / \mathrm{kg}\left({ }^{82} \mathrm{Rb}\right) \\
\text { or } 5 \mathrm{MBq} / \mathrm{kg} \\
\text { (ammonia) both } \\
\text { at stress and } \\
\text { rest }\end{array}$ & $\begin{array}{l}1053(+/-110) \\
\mathrm{MBq} \text { at stress } \\
\text { followed by } \\
1203(+/-\mathrm{MBq}) \\
\text { at rest }\end{array}$ & $\begin{array}{l}1000 \mathrm{MBq} \text { at rest } \\
\text { then } 1000 \mathrm{MBq} \text { at } \\
\text { stress }(2 \mathrm{~min} \\
\text { infusion at } 500 \\
\mathrm{MBq} / \mathrm{min})\end{array}$ \\
\hline Software & $\begin{array}{l}\text { 4-DM-Corridor } \\
\text { software } \\
\text { package }\end{array}$ & $\begin{array}{l}\text { Carimas } 2.4 \\
\text { software } \\
\text { (Turku, } \\
\text { Finland) }\end{array}$ & $\begin{array}{c}\text { FlowQuant } \\
\text { software }\end{array}$ & $\begin{array}{l}\text { PMOD software } \\
\text { package } \\
\text { (version 3.1; } \\
\text { PMOD } \\
\text { Technologies } \\
\text { Ltd.) }\end{array}$ & Not reported \\
\hline
\end{tabular}


Table 1 continued

\begin{tabular}{|c|c|c|c|c|c|}
\hline Study & $\begin{array}{l}\text { Giubbini } \\
\text { et } \text { al }^{13} \\
(2019)\end{array}$ & $\begin{array}{l}\text { Waterday } \\
\text { study } \\
\text { (2018) }\end{array}$ & $\begin{array}{l}\text { Wells et } \mathrm{al}^{7} \\
(2017)\end{array}$ & $\begin{array}{l}\text { Nkoulou } \\
\text { et } \mathrm{al}^{14}(2016)\end{array}$ & Ito et $\mathrm{al}^{8}(2003)$ \\
\hline $\begin{array}{l}\text { Comparison } \\
\text { to PET } \\
\text { mean } \\
\text { global } \\
\text { resting } \\
\text { MBF }\end{array}$ & $\begin{array}{l}\text { Similar with AC- } \\
\text { CZT and } \\
\text { different with } \\
\text { non-AC CZT }\end{array}$ & CZT higher & $\begin{array}{l}\text { Good correlation } \\
\text { of global MBF } \\
\text { measurements. }\end{array}$ & CZT similar & - \\
\hline $\begin{array}{l}\text { Comparison } \\
\text { to PET } \\
\text { mean } \\
\text { global } \\
\text { stress MBF }\end{array}$ & $\begin{array}{l}\text { AC CZT lower } \\
\text { Non-AC CZT } \\
\quad \text { higher }\end{array}$ & CZT higher & - & CZT lower & - \\
\hline $\begin{array}{l}\text { Comparison } \\
\text { to PET } \\
\text { mean } \\
\text { global } \\
\text { MFR }\end{array}$ & $\begin{array}{l}\text { Lower with both } \\
\text { AC and non- } \\
\text { AC CZT }\end{array}$ & Similar MFR & - & CZT lower MFR & SPECT lower \\
\hline
\end{tabular}

$M B F$, myocardial blood flow; $M F R$, Myocardial flow reserve; $B M I$, body mass index; $C A D$, Coronary artery disease; SPECT, Single positron emission tomography; $P E T$, positron emission tomography; $C Z T$, cadmium zinc telluride; $G E$, general electric; $D$, spectrum dynamics; $A C$, attenuation correction; $M B q$, mega becquerel

This study along with others paves the way for MBF quantification using SPECT with several caveats and questions, which require further exploration:

1. Standardization of protocols, radiotracers, acquisition, reconstruction, kinetic modeling, and postprocessing tools to reduce variability of $\mathrm{MBF}$ measurements.

2. Demonstration of intra- and inter-observer variabilities of these measurements or lack thereof in multicenter studies.

3. Assessment of variability introduced by the spatial resolution of SPECT, partial volume averaging, spillover, attenuation, motion and other artifacts while making these measurements.

4. Demonstration of the incremental value of SPECTderived MBF and possible ways to incorporate this information in making clinical decisions.

In conclusion, there is clearly a growing interest to evaluate the role of SPECT-derived absolute myocardial perfusion for diagnosis and prognosis. This study along with others will encourage further investigation towards standardization of MBF measurement by SPECT and understanding its clinical utility. Till that time caution should be exercised including SPECT MBF quantification in routine clinical practice.

\section{Disclosure}

Authors have no conflict of interest to declare.

\section{References}

1. Bengel FM. Leaving relativity behind: Quantitative clinical perfusion imaging. J Am Coll Cardiol 2011;58:749-51.

2. Bajaj NS, Osborne MT, Gupta A, Tavakkoli A, Bravo PE, Vita T, et al. Coronary microvascular dysfunction and cardiovascular risk in obese patients. J Am Coll Cardiol 2018;72:707-17.

3. Osborne MT, Bajaj NS, Taqueti VR, Gupta A, Bravo PE, Hainer J, et al. Coronary microvascular dysfunction identifies patients at high risk of adverse events across cardiometabolic diseases. J Am Coll Cardiol 2017;70:2835-7.

4. Bravo PE, Bergmark BA, Vita T, Taqueti VR, Gupta A, Seidelmann $S$, et al. Diagnostic and prognostic value of myocardial blood flow quantification as non-invasive indicator of cardiac allograft vasculopathy. Eur Heart J 2018;39:316-23.

5. Murthy VL, Bateman TM, Beanlands RS, Berman DS, BorgesNeto S, Chareonthaitawee $P$, et al. Clinical quantification of myocardial blood flow using PET: Joint position paper of the SNMMI Cardiovascular Council and the ASNC. J Nucl Med 2018;59:273-93.

6. Gupta A, Taqueti VR, van de Hoef TP, Bajaj NS, Bravo PE, Murthy VL, et al. Integrated noninvasive physiological assessment of coronary circulatory function and impact on cardiovascular mortality in patients with stable coronary artery disease. Circulation 2017;136:2325-36.

7. Wells RG, Marvin B, Poirier M, Renaud J, deKemp RA, Ruddy TD. Optimization of SPECT measurement of myocardial blood 
flow with corrections for attenuation, motion, and blood binding compared with PET. J Nucl Med 2017;58:2013-9.

8. Ito Y, Katoh C, Noriyasu K, Kuge Y, Furuyama H, Morita K, et al. Estimation of myocardial blood flow and myocardial flow reserve by $99 \mathrm{mTc}$-sestamibi imaging: comparison with the results of [15O] $\mathrm{H}_{2} \mathrm{O}$ PET. Eur J Nucl Med Mol Imaging 2003;30:281-7.

9. Hsu B, Chen FC, Wu TC, Huang WS, Hou PN, Chen CC, et al. Quantitation of myocardial blood flow and myocardial flow reserve with $99 \mathrm{mTc}$-sestamibi dynamic SPECT/CT to enhance detection of coronary artery disease. Eur J Nucl Med Mol Imaging 2014;41:2294-306.

10. Slomka PJ, Patton JA, Berman DS, Germano G. Advances in technical aspects of myocardial perfusion SPECT imaging. J Nucl Cardiol 2009; 16:255-76.

11. Bocher M, Blevis IM, Tsukerman L, Shrem Y, Kovalski G, Volokh L. A fast cardiac gamma camera with dynamic SPECT capabilities: Design, system validation and future potential. Eur J Nucl Med Mol Imaging 2010;37:1887-902.

12. Wells RG, Timmins R, Klein R, Lockwood J, Marvin B, deKemp RA, et al. Dynamic SPECT measurement of absolute myocardial blood flow in a porcine model. J Nucl Med 2014;55:1685-91.
13. Giubbini RBM, Durmo M, Bonacina M, Peli A, Faggiano IAD, Milan E, et al. Comparison between N13NH3-PET and 99mTcTetrofosmin CZT SPECT in the evaluation of absolute myocardial blood flow and flow reserve. J Nucl Cardiol 2019. https://doi.org/ 10.1007/s12350-019-01939-x.

14. Agostini D, Roule V, Nganoa C, Roth N, Baavour R, Parienti JJ, et al. First validation of myocardial flow reserve assessed by dynamic (99m)Tc-sestamibi CZT-SPECT camera: head to head comparison with (15)O-water PET and fractional flow reserve in patients with suspected coronary artery disease. The WATERDAY study. Eur J Nucl Med Mol Imaging 2018;45:1079-90.

15. Nkoulou R, Fuchs TA, Pazhenkottil AP, Kuest SM, Ghadri JR, Stehli J, et al. Absolute myocardial blood flow and flow reserve assessed by gated SPECT with cadmium-zinc-telluride detectors using 99mTc-Tetrofosmin: Head-to-head comparison with $13 \mathrm{~N}$ ammonia PET. J Nucl Med 2016;57:1887-92.

Publisher's Note Springer Nature remains neutral with regard to jurisdictional claims in published maps and institutional affiliations. 